\title{
IMPACT OF THE MAZE PROCEDURE ON THE STROKE RATE IN PATIENTS WITH ATRIAL FIBRILLATION
}

James L. Cox, MD

Niv Ad, MD

Terry Palazzo, RN, MS
Objective: The incidence of stroke associated with atrial fibrillation, even in high-risk patients, can be reduced significantly by adequate anticoagulation. However, anticoagulation does not abolish the stroke rate, and unfortunately only $\mathbf{4 0 \%}$ of patients with atrial fibrillation actually receive anticoagulant therapy, even in areas where adequate health care is available. Methods: During the past 11.5 years, we have performed the maze procedure for the treatment of medically refractory atrial fibrillation in 306 patients, 58 of whom presented with a history of having had a stroke $(n=40)$ or transient ischemic attack $(n=18)$ before surgery. All patients with atrial fibrillation are at an increased risk for these complications, but they are especially prevalent in those patients with previous thromboembolic events and those with other recognized risk factors. Results: Among the 306 patients who had surgery, there were only 2 perioperative strokes $(0.7 \%)$, and in the 265 patients followed for up to 11.5 years after the maze procedure, there has been only 1 late minor stroke that has now completely resolved. Conclusions: The ability of the maze procedure to decrease the risk of stroke associated with atrial fibrillation so dramatically is likely due to the restoring of sinus rhythm and atrial transport function in combination with surgical removal or obliteration of the left atrial appendage, where most thrombi associated with atrial fibrillation develop. (J Thorac Cardiovasc Surg 1999;118:833-40)
$\mathrm{R}$ ecent studies have documented that 2.2 million people in the United States have atrial fibrillation and that approximately 75,000 strokes occur in these patients each year. ${ }^{1,2}$ Anticoagulation with warfarin sodium (Coumadin) and, in some instances, with aspirin therapy decreases the incidence of stroke caused by atrial fibrillation. ${ }^{3-11}$ Unfortunately, although anticoagulation is beneficial, it does not abolish the risk of stroke associated with atrial fibrillation. Indeed, the incidence of stroke in patients with atrial fibrillation who undergo anticoagulation optimally with warfarin sodium remains between $2 \%$ and $5 \%$ per year, depending on the patient's associated risk factors. ${ }^{3-6,8,11}$

From the Department of Thoracic and Cardiovascular Surgery, Georgetown University School of Medicine, Washington, DC.

Read at the Seventy-ninth Annual Meeting of The American Association for Thoracic Surgery, New Orleans, La, April 18-21, 1999.

Received for publication April 22, 1999; revisions requested June 3, 1999; revisions received Aug 2, 1999; accepted for publication Aug 3, 1999.

Address for reprints: James L. Cox, MD, Professor and Chairman, Department of Thoracic and Cardiovascular Surgery, Georgetown University Medical Center, 4 PHC, 3800 Reservoir Rd, NW, Washington, DC 20017.

Copyright (C) 1999 by Mosby, Inc.

0022-5223/99 \$8.00+ $0 \quad \mathbf{1 2 / 6 / 1 0 1 9 1 2}$
Moreover, even with access to adequate medical care, only $40 \%$ of patients with atrial fibrillation are actually treated with anticoagulants. ${ }^{12,13}$ Thus even if one resides in an area with adequate medical services, the thromboembolic complications of atrial fibrillation remain a major health problem.

During the past 11.5 years, we have surgically ablated atrial fibrillation in a substantial number of patients who had prior strokes and transient ischemic attacks (TIAs) caused by left atrial mural thrombi associated with atrial fibrillation. These patients are at high risk for having a subsequent stroke even if they undergo anticoagulation. The fact that the remaining patients in our series also had atrial fibrillation means that they were also at a higher risk for having strokes than the general population. Our analysis indicates that surgical intervention with the maze procedure nearly eliminates the risk of stroke, even in patients who have had repeated episodes of thromboembolism related to atrial fibrillation preoperatively.

\section{Methods}

Between September 25, 1987, and March 25, 1999 (11.5 years), we performed the maze procedure in 306 patients for the treatment of atrial fibrillation. The first $32(10 \%)$ patients 
Table I. Postoperative anticoagulation regimen for patients undergoing the maze procedure for atrial fibrillation

\begin{tabular}{l}
\hline Permanent anticoagulation with warfarin sodium \\
All mitral valve replacements \\
Mechanical aortic valve replacements \\
Bentall procedures \\
Miscellaneous indications and combinations \\
No long-term anticoagulation with warfarin sodium \\
Temporary anticoagulation with warfarin sodium (78) \\
All patients with history of TE \\
Early patients in series \\
Miscellaneous indications \\
No postoperative anticoagulation with warfarin sodium (142) \\
Maze procedure only \\
Coronary artery bypass grafting \\
Mitral valve repair \\
Tissue aortic valve replacements \\
\hline
\end{tabular}

underwent the maze I procedure, ${ }^{14-17}$ the next $15(5 \%)$ patients underwent the maze II procedure, and the remaining 259 $(85 \%)$ patients underwent the maze III procedure. ${ }^{16-18}$ In the latter group 49 (19\%) of the 259 patients had the maze III procedure performed by using minimally invasive techniques.

Of the 306 patients who underwent the maze procedure for atrial fibrillation, $76 \%$ were men. Sixty-one percent of the patients had paroxysmal (intermittent) atrial fibrillation for an average of 8 years (range, $0.5-45$ years) before surgery. The other $39 \%$ of patients had chronic (continuous) atrial fibrillation for an average of 11 years (range, 0.3-39 years) before surgery. No patient had responded to medical therapy for atrial fibrillation, having been treated unsuccessfully with an average of 5.2 drugs per patient (range, 3-13 drugs). Surgical indications included arrhythmia intolerance in $75 \%$ of patients, drug intolerance in $6 \%$ of patients, and previous thromboembolic events in 19\% of patients. Twenty-four (8\%) patients had previous cardiac surgery, meaning that the maze procedure was performed as a reoperative procedure in these patients. One-hundred sixteen patients had 134 concomitant procedures along with the maze procedure, including mitral valve surgery (64 patients), aortic valve surgery (10 patients), coronary artery bypass surgery (41 patients), and procedures for other types of acquired and congenital cardiac anomalies (19 patients).

These surgical patients were divided into 2 groups: group 1 $(n=58)$ consisted of patients who had experienced at least 1 documented thromboembolic event before surgery, and group $2(n=248)$ consisted of patients who had not experienced any known episode of thromboembolism before surgery. The preoperative thromboembolic events that were experienced by the 58 patients in group 1 included frank stroke in 40 patients and TIAs in 18 patients. Twelve of the 40 patients who had a history of stroke had also undergone TIAs. Five of these 40 patients also had noncerebral thromboembolism before surgery. Two patients had over 5 separate strokes during the 2 weeks before surgery, and as a result, the maze procedure was performed as an urgent procedure after air ambulance evacuation to our hospital. Of the 18 patients who had TIAs, $80 \%$ experienced more than one such episode before surgery. Interestingly, despite the fact that all of the patients in group $1 \mathrm{had}$ experienced at least one episode of systemic thromboembolism associated with their atrial fibrillation, only $76 \%$ of them had undergone anticoagulation before surgery.

Postoperative anticoagulation regimen. Details of the postoperative anticoagulation regimen are presented in Table I.

Permanent anticoagulation with warfarin sodium $(n=45)$.

We do not give warfarin sodium to patients undergoing the maze procedure alone. All patients with any type of mitral valve prosthesis underwent anticoagulation unless there was a specific contraindication in a patient with a tissue mitral prosthesis. All patients receiving mechanical aortic valve prostheses, including the 3 Bentall procedures in this series, underwent permanent anticoagulation. Miscellaneous indications included various combinations of procedures in which one component of the procedure did not warrant permanent anticoagulation while another component did.

No long-term anticoagulation with warfarin sodium $(n=$ 220). The patients who did not undergo permanent anticoagulation after the maze procedure can be divided into 2 subgroups. The first subgroup $(n=78)$ underwent temporary anticoagulation for 3 months. This subgroup included all patients who had a history of having had a thromboembolic event preoperatively $(n=58)$. The decision regarding whether to use anticoagulants with these patients and for what period of time was entirely empirical. The reasons for temporarily using anticoagulants with the other 20 patients were varied. When we first began the series in 1987, we routinely used anticoagulants with all patients undergoing the maze procedure for 3 months postoperatively, but that practice was discontinued in 1991. Throughout the series there have been a few patients in whom early resistant perioperative atrial fibrillation has developed for whom we have elected to use anticoagulants. These patients have all converted out of atrial fibrillation before being discharged from the hospital, but we have elected to continue giving them warfarin sodium for 3 months. Thereafter they all continue taking aspirin unless there is a specific contraindication to doing so.

The second subgroup $(n=142)$ underwent no postoperative anticoagulation with warfarin sodium. This is the largest subgroup in the series because it includes all patients who underwent the maze procedure only, except for those with a history of previous thromboembolism. It also includes those patients who underwent the maze procedure combined with mitral valve repair, coronary artery bypass surgery, and tissue aortic valve replacements who had no other concomitant procedure that would have dictated the necessity for anticoagulation. These patients routinely received aspirin therapy unless there was a specific contraindication to doing so.

\section{Results}

Operative results. The overall operative mortality rate in this series of patients was 3.3\%. Only one peri- 
Volume 118, Number 5

Table II. Number of patients available for evaluation of late results

\begin{tabular}{|c|c|c|c|c|c|}
\hline & Total No. of patients & Operative deaths & Followed $<3$ mo & Late deaths & $\begin{array}{c}\text { Total followed } \\
\text { long-term }\end{array}$ \\
\hline Group 1 & 58 & 2 & 6 & 0 & 50 \\
\hline Group 2 & 248 & 8 & 19 & 6 & 215 \\
\hline Total & 306 & 10 & 25 & 6 & 265 \\
\hline
\end{tabular}

Table III. Stroke rate during the perioperative period and on late follow-up for patients undergoing the maze procedure

\begin{tabular}{lccc}
\hline & Group 1 & Group 2 & Total \\
\hline $\begin{array}{l}\text { Perioperative } \\
(<3 \text { mo })\end{array}$ & $1.7 \%(\mathrm{n}=58)$ & $0.4 \%(\mathrm{n}=248)$ & $0.7 \%(\mathrm{n}=306)$ \\
$\begin{array}{l}\text { Follow-up } \\
(3 \text { mo-11.5 } \mathrm{y})\end{array}$ & $0 \%(\mathrm{n}=50)$ & $0.5 \%(\mathrm{n}=215)$ & $0.4 \%(\mathrm{n}=265)$ \\
\hline
\end{tabular}

operative death occurred as a result of the maze procedure itself, a delayed tamponade 9 days after surgery. The remaining deaths occurred in patients undergoing concomitant surgery for associated cardiac conditions, including hypertrophic obstructive cardiomyopathy (2 patients), respiratory insufficiency caused by black lung disease and amiodarone toxicity (2 patients), doublevalve surgery (1 patient), redo double-valve surgery ( 1 patient), mitral valve combined with coronary artery bypass surgery ( 1 patient), redo mitral valve surgery (1 patient), and redo constrictive pericarditis surgery (1 patient). The most common perioperative complication after the maze procedure is the development of temporary postoperative atrial fibrillation in $42 \%$ of patients. Interestingly, this figure has dropped to $22 \%$ in the past year, probably because the majority $(70 \%)$ of the maze procedures are now performed by using minimally invasive techniques. As previously reported, the development of perioperative atrial fibrillation has no adverse effect on the long-term success rate of the maze procedure in curing atrial fibrillation. ${ }^{17-21}$

A total of 265 patients have been followed between 3 months and 11.5 years (mean, $3.7 \pm 2.9$ years) after surgery (Table II). After allowing each patient 3 months to heal from the operation, all antitachycardia medications (if they had been instituted postoperatively) were discontinued. Any subsequent documented episode of atrial flutter or atrial fibrillation was classified as a recurrence, even if it was only a single episode. According to this rather stringent criterion, the recurrence rate was $5 \%$ for all maze patients, but it was only $1.8 \%$ in the 222 patients who had undergone the maze III procedure more than 3 months previously. All
Table IV. Rate of TIAs during the perioperative period and on late follow-up for patients undergoing the maze procedure

\begin{tabular}{lccc}
\hline & Group 1 & Group 2 & Total \\
\hline $\begin{array}{l}\text { Perioperative } \\
(<3 \text { mo })\end{array}$ & $1.7 \%(\mathrm{n}=58)$ & $0.4 \%(\mathrm{n}=248)$ & $0.7 \%(\mathrm{n}=306)$ \\
$\begin{array}{l}\text { Follow-up } \\
(3 \text { mo-11.5 y })\end{array}$ & $2 \%(\mathrm{n}=50)$ & $0.5 \%(\mathrm{n}=215)$ & $0.8 \%(\mathrm{n}=265)$ \\
\hline
\end{tabular}

patients with recurrences were converted out of atrial fibrillation and have remained in a regular rhythm. All but 2 patients have required only one drug to maintain the regular rhythm. Thus at the time of this update, $95 \%$ of all patients have been cured of atrial fibrillation by the maze procedure alone, and the other $5 \%$ of patients have been cured with a combination of the maze procedure and postoperative antitachycardia drugs. After the maze III procedure, which has been used exclusively since April 1992, 98.2\% of patients have been cured with surgery alone, and only $1.8 \%$ have required postoperative antiarrhythmic medications. Currently, no patient who has undergone the maze procedure has either atrial fibrillation or atrial flutter.

Stroke rate in patients undergoing the maze procedure. Stroke rates in patients undergoing the maze procedure are shown in Table III. During the perioperative period, one patient in group $1(1.7 \%)$ and one patient in group $2(0.4 \%)$ had a stroke. In these 2 patients who had perioperative strokes, there remains a left foot drop in one patient at 9 years and mild residual weakness on the left side in the other at 5 months.

During the subsequent 11.5 years of follow-up, there were no strokes in group 1 patients and only one minor stroke in group 2 patients. Thus the overall stroke rate in all patients undergoing the maze procedure was $0.7 \%$ in the perioperative period and $0.4 \%$ in the follow-up period of 11.5 years.

TIA rate in patients undergoing the maze procedure. TIA rates in patients undergoing the maze procedure are shown in Table IV. During the perioperative period, one patient in group $1(1.7 \%)$ and one patient in group $2(0.4 \%)$ had a TIA. The 2 perioperative TIAs 


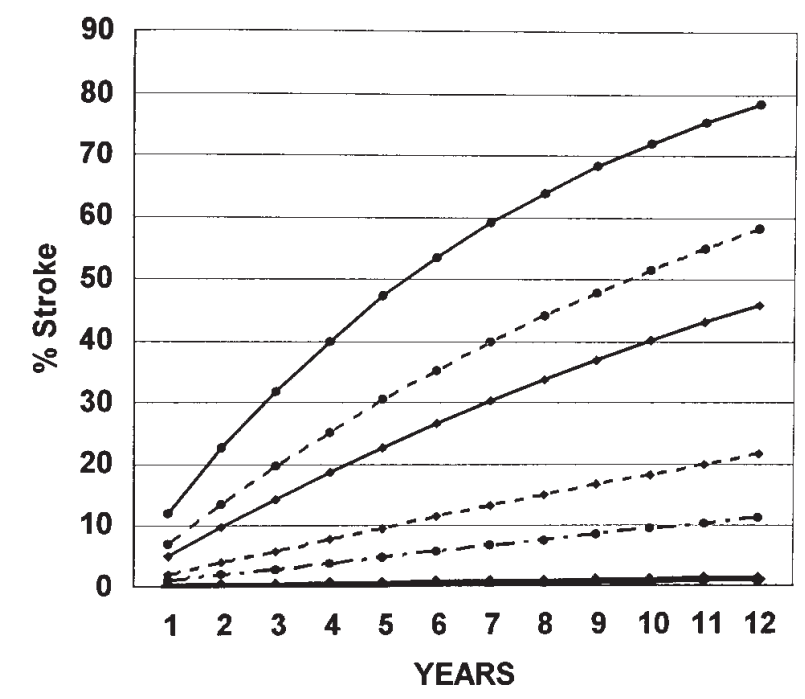

Fig 1. Stroke rate curves for patients with atrial fibrillation treated nonsurgically ${ }^{3-13}$ compared with patients with atrial fibrillation treated with the maze procedure. The curves are described below as beginning at the top curve and progressing downward to the bottom curve. Top curve, Atrial fibrillation, positive risk factors, previous thromboembolism, no anticoagulation; second curve, atrial fibrillation, positive risk factors, no previous thromboembolism, no anticoagulation; third curve, atrial fibrillation, positive risk factors, previous thromboembolism, anticoagulation; fourth curve, atrial fibrillation, positive risk factors, no previous thromboembolism, anticoagulation; fifth curve, atrial fibrillation, no risk factors, no previous thromboembolism, no anticoagulation; bottom curve, After the maze procedure.

were treated with anticoagulation for 3 months with no subsequent events.

During the subsequent 11.5 years of follow-up, there was one TIA in group 1 (2.0\%) and one TIA in group 2 $(0.5 \%)$. The 2 patients who had late TIAs were immediately anticoagulated and have remained so now for up to 8 years. Neither patient has had any further thromboembolic events. Thus the TIA rate in all patients undergoing the maze procedure was $0.7 \%$ in the perioperative period and $0.8 \%$ in the follow-up period of 11.5 years.

\section{Analysis of results by clinical category}

Atrial fibrillation with no associated risk factors. This group includes lone atrial fibrillation, a term that generally refers to patients who have atrial fibrillation with no other detectable cardiac abnormalities, but it may also include patients with associated cardiac anomalies that do not represent positive risk factors for stroke. The stroke rate in this group of patients is only $1 \%$ per year, even without anticoagulation. ${ }^{8,11}$ It is for
Table V. Perioperative stroke rates for the major categories of cardiac surgical procedures

\begin{tabular}{lccc}
\hline Surgical procedure & $\begin{array}{c}\text { Percent } \\
\text { permanent } \\
\text { stroke }\end{array}$ & $\begin{array}{c}\text { Percent } \\
\text { transient } \\
\text { stroke }\end{array}$ & $\begin{array}{c}\text { Percent } \\
\text { total } \\
\text { strokes }\end{array}$ \\
\hline CABG + MVR & $4.17 \%$ & $1.73 \%$ & $5.90 \%$ \\
CABG + AVR & $3.40 \%$ & $1.60 \%$ & $5.00 \%$ \\
CABG + MV repair & $3.12 \%$ & $1.41 \%$ & $4.53 \%$ \\
AVR + MVR & $2.73 \%$ & $1.51 \%$ & $4.24 \%$ \\
MV repair & $1.85 \%$ & $1.45 \%$ & $3.30 \%$ \\
MVR & $2.28 \%$ & $0.98 \%$ & $3.26 \%$ \\
AVR & $1.73 \%$ & $1.23 \%$ & $2.96 \%$ \\
CABG & $1.69 \%$ & $0.75 \%$ & $2.44 \%$ \\
Maze \pm other & $0.65 \%$ & $0 \%$ & $0.65 \%$ \\
\hline
\end{tabular}

$C A B G$, Coronary artery bypass grafting; $M V R$, mitral valve replacement; $A V R$, aortic valve replacement; $M V$, mitral valve.

this reason that these patients are not usually anticoagulated in clinical practice.

In our series 162 patients corresponded to this category, and surprisingly, $81(50 \%)$ of them had undergone anticoagulation at the time they presented to us. After the maze procedure, none of these patients received long-term anticoagulation, and only one minor stroke occurred during the follow-up period of $3.8 \pm 3.0$ years.

Atrial fibrillation with associated risk factors but no previous stroke or TIA. Several studies have documented the major risk factors associated with an increased likelihood of having a stroke caused by atrial fibrillation. These include advanced age, hypertension, diabetes mellitus, previous stroke or TIA, congestive heart failure, and ischemic heart disease. ${ }^{8,11}$ The stroke rate associated with one or more of these risk factors and atrial fibrillation is $6 \%$ to $8 \%$ per year in patients not undergoing anticoagulation and $2 \%$ per year in patients undergoing adequate anticoagulation. In our series 86 patients were in this category, and they had a follow-up period of $3.1 \pm 2.9$ years. Forty-six percent of these patients underwent anticoagulation preoperatively, and $54 \%$ did not undergo anticoagulation. This pattern of anticoagulation in these high-risk patients is consistent with previous reports indicating that only $40 \%$ of such patients actually receive anticoagulation. ${ }^{12,13}$ None of our patients in this category had strokes postoperatively.

Atrial fibrillation with associated risk factors including previous stroke or TIA. The incidence of stroke in patients in this category is $12 \%$ per year if the patient has not undergone anticoagulation ${ }^{8,9,11}$ and $5.1 \%$ per year if the patient has undergone anticoagulation. ${ }^{8}$ In our series 58 patients corresponded to this category. Forty-four patients had undergone anticoagulation, and 
14 patients had not undergone anticoagulation at the time they presented to us. The follow-up period for this group of patients, in which no postoperative strokes occurred, was $3.7 \pm 2.5$ years.

\section{Discussion}

Perioperative stroke rate. One of the major concerns when we first began to perform the maze procedure clinically in 1987 was that the incidence of perioperative stroke in these patients, many of whom had already had a thromboembolic event, would be substantially higher than that associated with other types of cardiac surgery. Much to our surprise, the exact opposite has been the case (Table V). The 1998 data from the National Database for Adult Cardiac Surgery published by the Society of Thoracic Surgeons document that the perioperative stroke rate is higher for every major cardiac surgery procedure than it is for the maze procedure. ${ }^{22}$

One explanation for the paradox of having the lowest perioperative stroke rate in patients who might be expected to have the highest is that the average age of the patients in this series was substantially less than the average age of patients in most reported cardiac surgical series. It is our suspicion that in addition to the age factor, the absence of a left atrial appendage postoperatively probably contributes to the low perioperative stroke rate.

Long-term postoperative stroke rate. Follow-up in these patients is an ongoing project and is accomplished either by direct patient contact or through contact with one of the referring physicians. The maze procedure not only reduced the rate of stroke in all categories of patients but nearly abolished the risk of stroke postoperatively (Fig 1). By using the published stroke rates for the various categories of patients, it would appear that at a mean of 3.9 years after surgery, the maze procedure has thus far prevented strokes that would have occurred in approximately 29 patients had the surgical procedure not been applied.

To understand why the maze procedure is so effective in reducing the incidence of stroke associated with atrial fibrillation, it is helpful to review some of the salient points about thromboembolism associated with atrial fibrillation. It is sometimes not appreciated that both atria are fibrillating during atrial fibrillation. This raises the question as to why systemic embolization from thrombi formed in the left atrium are so much more common than pulmonary embolization from thrombi that might logically form in a similar manner in the right atrium. Clinical experience, however, documents that although left atrial thrombi are a common occur- rence in atrial fibrillation, right atrial thrombi are not. Differences in the right atrial and left atrial anatomy probably explain these clinical patterns. For example, the orifice of the right atrial appendage is so broadbased that it is not usually thought of as being an orifice at all. Moreover, this wide-open entrance into the relatively shallow right atrial appendage is located immediately adjacent to the stream of blood flow from the superior vena cava into the right atrium and across the tricuspid valve. Therefore there is little opportunity for stasis of blood within the body of the right atrial appendage, even when the atrium is fibrillating. On the contrary, the orifice of the left atrial appendage is an identifiable anatomic opening in virtually all patients. This narrow orifice leads into a relatively long narrow lumen of the left atrial appendage, which is eccentric to the stream of blood flow from the pulmonary veins to the mitral valve. Therefore when the atrium is fibrillating, severe stasis of blood in the left atrial appendage is virtually assured and is one of the major factors resulting in thrombus formation in the left atrial appendage.

Perhaps the major reason for the maze procedure's protective effect against thromboembolism is that it cures the atrial fibrillation. Our studies have documented that postoperatively the left atrium functions well in 93\% of patients and the right atrium functions essentially normally in $98 \%$ of patients. ${ }^{21}$ This restoration of atrial contractile function in both atria in over $90 \%$ of patients is clearly an important factor in reducing postoperative stasis of blood flow and hence in reducing the likelihood of the formation of atrial thrombi. Undoubtedly, another reason for the absence of strokes after the maze procedure is the fact that the left atrial appendage is either excised or its orifice is surgically closed as a part of the surgical procedure. This means that after the maze procedure, there is no atrial fibrillation, there are no atrial appendages, and the atria are contracting. Thus there is no reason for atrial thrombi to form unless there is an underlying hypercoagulability syndrome in which case the surgically altered left atrium should theoretically be no more likely to harbor mural thrombi than any other nearby structure, such as the pulmonary veins or the left ventricle.

Another question is whether the $7 \%$ of patients with no demonstrable left atrial contractile function after the maze procedure should undergo anticoagulation. We have not done so for the following reasons. All of these patients have essentially normal function of the right atrium and normal right atrial to right ventricular synchrony. This results in a normal right-sided cardiac output that is delivered to the left side of the heart through the pulmonary circulation. In the presence of a reason- 
ably normal left ventricle, studies have shown that it makes no difference whether the left atrium is contracting because the left ventricle will immediately adapt to the normal right-sided output that has just been delivered to it. ${ }^{23}$ The volume of flow through the left atrium remains normal, the left ventricular filling is regular, and there is no left atrial appendage to serve as a nidus for thrombus formation. Thus there is no reason to suspect that systemic thromboembolism would be any higher in these patients than it is in those patients with demonstrable left atrial contraction. This is also the reason why we have never placed any particular importance on the magnitude of left atrial contraction after the maze procedure, although it is clearly more esthetically pleasing to have it present postoperatively.

In summary, the maze procedure has proven to be very effective in abolishing the threat of a stroke associated with atrial fibrillation without the need for anticoagulation. The data presented in this study raise the question of whether the surgical indications for the maze procedure should be expanded to include the prevention of stroke.

\section{REFERENCES}

1. Freinberg WM, Blackshear JL, Laupacis A, Kromnal R, Hart RG. Prevalence, age, distribution and gender of patients with atrial fibrillation. Arch Intern Med 1995;155:469-73.

2. Wolf PA, Mitchel JB, Baker CS, Kannel WB, D'Agostino RB. Impact of atrial fibrillation on mortality stroke, and medical cost. Arch Intern Med 1998;158:229-34.

3. Petersen P, Boysen G, Godtfredsen J, Andersen E, Andersen B. Placebo-controlled, randomised trial of warfarin and aspirin for prevention of thromboembolic complications in chronic atrial fibrillation: The Copenhagen AFASAK study. Lancet 1989;1:175-8.

4. The Boston Area Anticoagulation Trial for Atrial Fibrillation Investigators. The effect of low dose warfarin on the risk of strokes in patients with non-rheumatic atrial fibrillation. N Engl J Med 1990;323:1505-11.

5. Stroke Prevention in Atrial Fibrillation Investigators. Stroke prevention in atrial fibrillation study: final results. Circulation 1991; 84:527-39.

6. Connolly S, Laupacic A, Gent M. Candian atrial fibrillation anticoagulation (CAFA) study. J Am Coll Cardiol 1991;18:349-55.

7. Stroke Prevention in Atrial Fibrillation Investigators. Warfarin versus aspirin for prevention of thromboembolism in atrial fibrillation: Stroke Prevention in Atrial Fibrillation II study. Lancet 1994;343:687-91.

8. Risk factors for stroke and efficacy of antithrombotic therapy in atrial fibrillation. Analysis of pooled data from five randomized controlled trials. Arch Intern Med 1994;154:1449-57.

9. Albers GW. Atrial fibrillation and stroke: three new studies, three remaining questions. Arch Intern Med 1998;158:1487-91.

10. EAFT (European Atrial Fibrillation Trial) Study Group. Secondary prevention in non-rheumatic atrial fibrillation after transient ischaemic attack or minor stroke. Lancet 1993;342: 1255-62.
11. Hart RG, Sherman DG, Easton JD, Cairns JA. Prevention of stroke in patients with nonvalvular atrial fibrillation. Neurology 1998;51:674-81.

12. Brass LM, Krurnholz HM, Scino JM, Radford M. Warfarin use among patients with atrial fibrillation. Stroke 1997;28:2382-9.

13. Stafford RS, Singer DE. Recent national patterns of warfarin use in atrial fibrillation. Circulation 1987;97:1231-3.

14. Cox JL, Canavan TE, Schuessler RB, Cain ME, Lindsay BD, Stone C, et al. The surgical treatment of atrial fibrillation. II. Intraoperative electrophysiologic mapping and description of the electrophysiologic basis of atrial flutter and atrial fibrillation. J Thorac Cardiovasc Surg 1991;101:406-26.

15. Cox JL, Schuessler RB, D'Agostino HJ, Stone C, Chang BC, Cain ME, et al. The surgical treatment of atrial fibrillation. III. Development of a definitive surgical procedure. J Thorac Cardiovasc Surg 1991;101:569-83.

16. Cox JL. The surgical treatment of atrial fibrillation. IV. Surgical technique. J Thorac Cardiovasc Surg 1991;101:584-92.

17. Cox JL, Boineau JP, Scheussler RB, Ferguson TB Jr, Cain ME, Lindsay BD, et al. Successful surgical treatment of atrial fibrillation. JAMA 1991;266:1976-80.

18. Cox JL, Boineau JP, Scheussler RB, Lappas DG. Modification of the maze procedure for atrial flutter and atrial fibrillation. I. Rationale and surgical results. J Thorac Cardiovasc Surg 1995; 110:473-84.

19. Cox JL, Jaquiss RI, Schuessler RB, Boineau IP. Modification of the maze procedure for atrial flutter and atrial fibrillation. II. Surgical technique of the maze III Procedure. J Thorac Cardiovasc Surg 1995;110:485-95.

20. Cox JL, Boineau JP, Scheussler RB, Kater KM, Lappas DG. Five-year experience with the maze procedure for atrial fibrillation. Ann Thorac Surg 1994;56:814-24.

21. Cox JL, Scheussler RB, Lappas DG, Boineau JP. An 8.5-year clinical experience with surgery for atrial fibrillation. Ann Surg 1996;224:267-75.

22. Society of Thoracic Surgeons. National adult cardiac surgery database. Society of Thoracic Surgeons; 1998.

23. Williams JM, Ungerleider RM, Lofland GK, Cox JL. Left atrial isolation: new technique for the treatment of supraventricular arrhythmias. J Thorac Cardiovasc Surg 1980;80:373-83.

\section{Discussion}

Dr Hartzell V. Schaff (Rochester, Minn). This follow-up of 306 patients focuses on late stroke but also provides some important information on the persistent benefit of the maze procedure in eliminating the recurrence of atrial fibrillation in a follow-up that extends to 11 years.

The report of only 2 perioperative neurologic events, a risk of $0.7 \%$, and one late stroke is striking. In his article Dr Cox stratifies this large patient group according to preoperative clinical characteristics to provide some yardstick against which surgical results can be measured. One hundred sixtytwo $(53 \%)$ patients had paroxysmal or chronic atrial fibrillation with no additional risk factors, and as mentioned, the risk of stroke in this group is so low that anticoagulation is not necessary. Therefore clearly the maze procedure would not be indicated. Eighty-six (28\%) patients had no previous cerebrovascular accidents but were considered at high risk because of the presence of additional risk factors, and the 
question of whether the maze procedure might be indicated to prevent stroke in this group is still unsettled.

Our own experience supports the conclusion that the maze procedure does reduce the risk of thromboembolic stroke, and over a 6-year period, from 1993 through 1998, we performed the operation in 212 patients. There have been 2 deaths early after surgery, yielding a perioperative mortality rate of approximately $1 \%$, and atrial fibrillation was abolished in $90 \%$ of patients.

Our experience differs somewhat from that of Dr Cox in that $76 \%$ of our patients had concomitant procedures, and $70 \%$ of these were mitral valve repairs. In an effort to learn whether the addition of the maze procedure to the mitral valve repair reduced risk of postoperative stroke, we compared 39 patients who had the combined operation to 58 patients who had mitral valve repair with atrial fibrillation.

During a follow-up period extending to 3 years, 4 patients in the control group had a stroke or TIA, and 4 patients had bleeding complications caused by warfarin sodium. The difference in the risk of stroke approached statistical significance, but more important, when we combine the end point of stroke and bleeding caused by anticoagulation, the reduction in events was statistically significant. We believe that the restoration of sinus rhythm also protects the patients from the hazards of bleeding and is an important issue.

Dr Cox, I have several questions. The perioperative stroke rate of $0.7 \%$ is lower than one would expect, as you point out, for most surgical procedures, and yet over $40 \%$ of the patients had recurrence of atrial fibrillation. This suggests that in addition to the relatively young age, obliteration of the left atrial appendage is an important mechanism in stroke reduction. I suppose if we carry this assumption to its logical conclusion, the question arises as to whether every patient undergoing cardiac surgery should have ligation of the left atrial appendage, and I would appreciate your comments on this.

Along the same lines, you detail the protocol for anticoagulation but did not mention antiplatelet therapy. Do you use aspirin in the patients early, and if so, do you continue it?

If the operation can be performed at low risk, it surely seems reasonable to recommend it for patients with prior TIAs and thromboembolic events, especially those requiring warfarin sodium for anticoagulation, but for patients without preoperative neurologic events, the presence of which risk factors for stroke would persuade you to advise a maze operation?

Finally, you emphasize the recent development of minimally invasive surgery, and as I understand your procedure, interruption of the left atrial conduction pathway is accomplished by cryolesions. We have noticed, in postoperative echocardiographic studies, a fairly uniform reduction in left atrial size caused by simple incision and suture. Do you believe that reducing the size of the left atrium is an important byproduct of the procedure, and if so, do you exclude patients from minimally invasive procedures who have enlarged left atria?

Dr Cox. Thank you, Dr Schaff. As usual, I expected and received a very erudite discussion of this problem, and I appreciate your comments very much.

In terms of the perioperative stroke rate, I do believe that the fact that these patients were younger is important. However, I also believe that ligation of the left atrial appendage is critical in preventing early and late strokes in these patients. Whether we should do this on all patients is questionable, but a prospective study of, for example, patients undergoing coronary bypass, in whom left atrial ligation is prospectively randomized, seems to me to be a reasonable proposition.

Your question also raises the issue of how many perioperative strokes are caused by atrial fibrillation as opposed to atherosclerosis of the ascending aorta or other causes. I believe that atherosclerosis is the major cause of strokes in cardiac surgery patients. However, Dr Larry Creswell wrote a paper a few years ago from our group in St Louis showing that the perioperative stroke rate is higher in patients with postoperative atrial fibrillation. Nevertheless, before I would recommend routine atrial appendage ligation in all cardiac surgery patients, I would prefer to see a prospective randomized trial.

In answer to your second question regarding aspirin use, we routinely give aspirin to these patients postoperatively if they do not require warfarin sodium for other reasons, such as mechanical valve replacement. I did not mention the effects of aspirin on the incidence of stroke, but as you know, it also has a beneficial effect in some patients. Whether we keep those patients on aspirin beyond 3 months is really left to the patient. We do not require that they continue aspirin beyond that time.

Your next question related to the importance of reducing the size of the left atrium. I do not think that left atrial size is important if one performs the standard maze procedure in which multiple incisions are made because one can trim the atrium down in size before closing the incisions. In addition, even though the minimally invasive approach that we now use routinely requires fewer incisions, I would not exclude someone from minimally invasive surgery on the basis of a large atrium. If we have a patient with a large left atrium, I extend the length of the incision between the pulmonary veins and the mitral valve much further into the left atrium than normal and then trim each side of the incision before closing. The optimal place to decrease the size of the large left atrium is between the pulmonary veins and the mitral valve anulus.

Finally, you asked a question about which risk factors other than thromboembolism I would include as indications for performing the maze procedure. If a patient has one or two of the risk factors and has undergone adequate anticoagulation but has had atrial fibrillation for several years, the risk of stroke becomes formidable. For example, if a patient has positive risk factors and no previous history of thromboembolism but then has a TIA, that patient must undergo anticoagulation. Even with anticoagulation, $50 \%$ of those patients will have a stroke in 12 years, which was the duration of this study. Thus if a patient has had atrial fibrillation for 6 or 7 years and is worried about a stroke, even though 
otherwise normal, I would consider performing a minimally invasive maze procedure in view of the results presented today. I would hasten to add, however, that in patients with lone atrial fibrillation who are at low risk of having a stroke, we do not perform the maze procedure because of the threat of a stroke.

Your comment about considering both bleeding from warfarin sodium and thromboembolic stroke in analyses such as this is a very important one. We elected not to confuse the issue of thromboembolic strokes with hemorrhagic strokes caused by anticoagulation in this study. Clearly, including the incidence of hemorrhagic strokes in patients receiving warfarin sodium would have increased all the numbers in this study and would have made an even stronger argument for permanently ablating atrial fibrillation and the risk of stroke, even if it means undergoing a surgical procedure.

\section{ON THE MOVE?}

Don't miss a single issue of the journal! To ensure prompt service when you change your address, please photocopy and complete the form below.

Please send your change of address notification at least six weeks before your move to ensure continued service. We regret we cannot guarantee replacement of issues missed due to late notification.

\section{JOURNAL TITLE:}

Fill in the title of the journal here.

\section{OLD ADDRESS:}

Affix the address label from a recent issue of the journal here.

\section{NEW ADDRESS: \\ Clearly print your new address here.}

Name

Address

City/State/ZIP
COPY AND MAIL THIS FORM TO:

Periodical Subscription Services

Mosby, Inc.

11830 Westline Industrial Dr.

St. Louis, MO 63146-3318
OR FAX TO:

314-432-1158

N/ Mosby
OR PHONE:

1-800-453-4351

Outside the U.S., call

314-453-4351 